\title{
Two Remarks on Skew Tableaux
}

\author{
Richard P. Stanley* \\ Department of Mathematics \\ Massachusetts Institute of Technology \\ Cambridge, MA 02139 \\ rstan@math.mit.edu
}

Submitted: Feb 22, 2011; Accepted: Jun 27, 2011; Published: Jul 15, 2011

Mathematics Subject Classification: 05 E05

\begin{abstract}
This paper contains two results on the number $f^{\sigma / \tau}$ of standard skew Young tableaux of shape $\sigma / \tau$. The first concerns generating functions for certain classes of "periodic" shapes related to work of Gessel-Viennot and Baryshnikov-Romik. The second result gives an evaluation of the skew Schur function $s_{\lambda / \mu}(x)$ at $x=$ $\left(1,1 / 2^{2 k}, 1 / 3^{2 k}, \ldots\right)$ for $k=1,2,3$ in terms of $f^{\sigma / \tau}$ for a certain skew shape $\sigma / \tau$ depending on $\lambda / \mu$.
\end{abstract}

\section{Introduction}

We assume familiarity with the basic theory of symmetric functions and tableaux from $[6$, Chap. 7]. Baryshnikov and Romik [1] obtain explicit formulas for the number of standard Young tableaux of certain skew shapes which they call diagonal strips. As the strips become thicker, their formulas becomes more and more complicated. In the next section we give simple generating functions for certain classes of diagonal strips of arbitrarily large thickness. Our results are neither a subset nor superset of those of Baryshnikov and Romik. Our results in Section 2 were also obtained in unpublished work of Gessel and Viennot [3, $§ 11]$, but our approach is more straightforward.

In Section 3 we obtain a formula for the evaluation of skew Schur functions $s_{\lambda / \mu}(x)$ at $x=\left(1,1 / 2^{2 k}, 1 / 3^{2 k}, \ldots\right)$ for $k=1,2,3$. These formulas have the following form. Let $n=|\lambda / \mu|$ (the number of squares in the diagram of $\lambda / \mu$ ), and choose $m \geq \lambda_{1}$. Then

$$
s_{\lambda / \mu}\left(1,1 / 2^{2 k}, 1 / 3^{2 k}, \ldots\right)=c(n, m) f^{\sigma / \tau} \pi^{2 n},
$$

*This author's contribution is based upon work supported by the National Science Foundation under Grant No. 0604423. 
where $\sigma / \tau$ is a certain skew shape depending on $\lambda / \mu$ and $m$. Moreover, $f^{\sigma / \tau}$ denotes the number of standard Young tableaux of shape $\sigma / \tau$ and $c(n, m)$ is a simple explicit function. The special case $\sigma / \tau=(1)$ (the unique partition of 1 ) gives the Riemann zeta function evaluations $\zeta(2)=\pi^{2} / 6, \zeta(4)=\pi^{4} / 90$, and $\zeta(6)=\pi^{6} / 945$. The main tool for our results on both diagonal strips and $s_{\lambda / \mu}\left(1,1 / 2^{2 k}, 1 / 3^{2 k}, \ldots\right)$ is the Jacobi-Trudi identity and its dual version.

The computation of $s_{\lambda / \mu}\left(1,1 / 2^{2 k}, 1 / 3^{2 k}, \ldots\right)$ for all $\lambda$ and $\mu$ is equivalent to the computation of certain multiple zeta values. As explained in Section 3, in principle there is no problem in computing this very special case of multiple zeta values. Our contribution is to give an explicit formula involving skew Schur functions when $k=1,2,3$.

Let us recall the Jacobi-Trudi identity and its connection with skew SYT. Write $\ell(\lambda)$ for the length (number of parts) of $\lambda$. If $\ell(\lambda) \leq m$ and $\mu \subseteq \lambda$, then the Jacobi-Trudi asserts that

$$
s_{\lambda / \mu}=\operatorname{det}\left[h_{\lambda_{i}-\mu_{j}-i+j}\right]_{i, j=1}^{m},
$$

where $h_{r}$ denotes a complete symmetric function (with $h_{0}=1$ and $h_{r}=0$ for $r<0$ ). Let $|\lambda / \mu|=n$. It is an immediate consequence of the combinatorial definition of $s_{\lambda / \mu}[6$, Def. 7.10.1] that $f^{\lambda / \mu}$ is the coefficient of $x_{1} x_{2} \cdots x_{n}$ in $s_{\lambda / \mu}$. By taking the coefficient of $x_{1} x_{2} \cdots x_{n}$ in equation (1.1), or equivalently by applying the exponential specialization $[6, \S 7.8]$, we obtain the well-known formula [6, Cor. 7.16.3] of Aitken:

$$
f^{\lambda / \mu}=n ! \operatorname{det}\left[1 /\left(\lambda_{i}-\mu_{j}-i+j\right) !\right]_{i, j=1}^{m} .
$$

Similarly for $\lambda_{1} \leq m$ we have the dual Jacobi-Trudi identity

$$
s_{\lambda / \mu}=\operatorname{det}\left[e_{\lambda_{i}^{\prime}-\mu_{j}^{\prime}-i+j}\right]_{i, j=1}^{m},
$$

where $e_{r}$ denotes an elementary symmetric function (with $e_{0}=1$ and $e_{r}=0$ for $r<0$ ) and ' denotes the conjugate partition.

\section{Diagonal strips}

We begin with some connections between generating functions and determinants.

Lemma 2.1. Let $a_{i}, i \in \mathbb{Z}$, be elements of some commutative ring with 1 , with $a_{0}=1$ and $a_{i}=0$ for $i<0$. Let $f(k)=\operatorname{det}\left[a_{j-i+1}\right]_{1}^{k}$. In particular, $f(0)=1$. Then

$$
\sum_{k \geq 0} f(k) x^{k}=\frac{1}{1-a_{1} x+a_{2} x^{2}-\cdots} .
$$

Proof. This is a well-known result, implicit in [5, Prop. 2.6]. The terms in the expansion of the determinant coincide with those in the expansion of

$$
\frac{1}{1-a_{1} x+a_{2} x^{2}-\cdots}=1+F(x)+F(x)^{2}+\cdots,
$$

where $F(x)=a_{1} x-a_{2} x^{2}+\cdots$. 


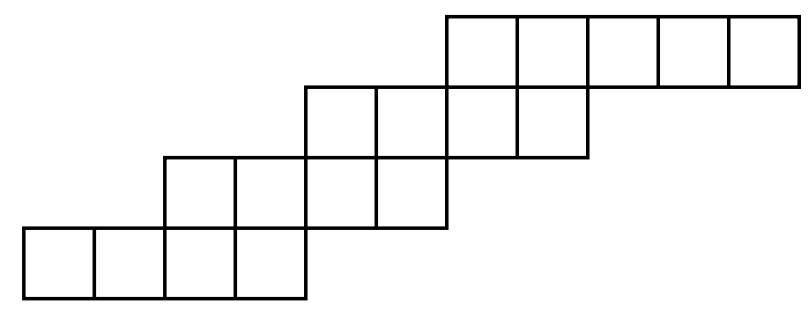

Figure 1: The partition $\sigma(5,4,3,4)$

Corollary 2.2. Suppose that in Lemma 2.1 we drop the condition $a_{0}=1$, say $a_{0}=\alpha$. Then

$$
\sum_{k \geq 0} f(k) x^{k}=\frac{1}{1+\sum_{i \geq 1}(-1)^{i} \alpha^{i-1} a_{i} x^{i}} .
$$

Proof. The case $\alpha=0$ is clear (and uninteresting), where we interpret $0^{0}=1$ for the summand indexed by $i=1$, so assume $\alpha \neq 0$. Let $A_{k}=\left[a_{j-i+1}\right]_{1}^{k}$, and let $D$ be the diagonal matrix $\operatorname{diag}\left(\alpha, \alpha^{2}, \ldots, \alpha^{k}\right)$. Then $D^{-1} A D=\left[\alpha^{j-i} a_{j-i+1}\right]$. Note that the $(j+1, j)$ entry is equal to 1 . Since $\operatorname{det} A=\operatorname{det} D^{-1} A D$, the proof follows from Lemma 2.1.

Corollary 2.3. Suppose that in Corollary 2.2 we let the first row of the matrix be arbitrary, i.e., let $M_{k}=\left(m_{i j}\right)_{1}^{k}$ be the $k \times k$ matrix defined by

$$
\begin{aligned}
m_{1 j} & =b_{j} \\
m_{i j} & =a_{j-i+1}, i \geq 2,
\end{aligned}
$$

where $a_{0}=\alpha$ and $a_{i}=0$ for $i<0$. Let $g(k)=\operatorname{det} M_{k}$. Then

$$
\sum_{k \geq 1} g(k) x^{k}=\frac{\sum_{j \geq 1}(-1)^{j-1} \alpha^{j-1} b_{j} x^{j}}{1+\sum_{i \geq 1}(-1)^{i} \alpha^{i-1} a_{i} x^{i}}
$$

Proof. If we remove the first row and $i$ th column from $M$, then we obtain a matrix $M_{i}=\left[\begin{array}{cc}B & C \\ 0 & A_{n-k}\end{array}\right]$, where $B$ is an upper triangular $(i-1) \times(i-1)$ matrix with $\alpha$ 's on the diagonal. Hence when we expand $\operatorname{det} M$ along the first row, we get

$$
\operatorname{det} M=b_{1}\left(\operatorname{det} A_{k-1}\right)-\alpha b_{2}\left(\operatorname{det} A_{k-2}\right)+\cdots+(-1)^{k-1} \alpha^{k-1} b_{k}\left(\operatorname{det} A_{0}\right) .
$$

The proof therefore follows from Corollary 2.2.

We now define the class of skew partitions of interest to us. For positive integers $a, b, c, n$, define $\sigma(a, b, c, n)$ to be the skew partition whose Young diagram has $a$ squares in the first row, $b$ squares in the other (nonempty) rows, and $n$ rows in all. Moreover, each row begins $c-1$ columns to the left of the row above, with $b \geq c$. For instance, Figure 1 shows the shape $\sigma(5,4,3,4)$. Thus $s_{\sigma(a, b, c, n)}$ denotes the Schur function of shape $\sigma(a, b, c, n)$. As above, let $h_{m}$ denote the $m$ th complete symmetric function. 
Theorem 2.4. Fix $a, b, c$ with $c \leq b<2 c$. Then

$$
\sum_{n \geq 1} s_{\sigma(a, b, c, n)} x^{n}=\frac{\sum_{n \geq 1}(-1)^{n-1} h_{b-c}^{n-1} h_{a+(n-1) c} x^{n}}{1-\sum_{n \geq 1}(-1)^{n-1} h_{b-c}^{n-1} h_{b+(n-1) c} x^{n}} .
$$

Proof. The Jacobi-Trudi matrix (1.1) has the form of Corollary 2.3 with $m_{1 j}=h_{a+(j-1) b}$, $a_{i}=h_{b+(i-1) c}$. In particular, $\alpha=a_{0}=b-c$. The condition $b<2 c$ is equivalent to $a_{i}=0$ for $i<0$. Hence the proof follows from Corollary 2.3.

Applying the exponential specialization, or just using Aitken's formula (1.2) directly, yields the following corollary.

Corollary 2.5. Fix $a, b, c$ with $c \leq b<2 c$. Then

$$
\sum_{n \geq 1} f^{\sigma(a, b, c, n)} \frac{x^{n}}{(a+(n-1) b) !}=\frac{\sum_{n \geq 1}(-1)^{n-1} \frac{x^{n}}{(b-c) !(b+(n-1) c) !}}{1-\sum_{n \geq 1}(-1)^{n-1} \frac{x^{n}}{(b-c) !(a+(n-1) c) !}} .
$$

For example, setting $(a, b, c)=(5,4,3)$ gives

$$
\sum_{n \geq 1} f^{\sigma(5,4,3, n)} \frac{x^{n}}{(4 n+1) !}=\frac{\sum_{n \geq 1}(-1)^{n-1} \frac{x^{n}}{(3 n+2) !}}{1-\sum_{n \geq 1}(-1)^{n-1} \frac{x^{n}}{(3 n+1) !}} .
$$

Note the curious appearance of the denominators $(4 n+1) !,(3 n+2)$ !, and $(3 n+1)$ !. It is unusual to have exponential generating function identities with $(a n+b)$ ! and $(c n+d)$ ! in the denominators, where one of $a$ and $c$ does not divide the other.

Note the special case $\sigma(3,3,2, n)$. Corollary 2.5 can be written in the form

$$
\sum_{n \geq 0} f^{\sigma(3,3,2, n)} \frac{x^{2 n}}{(3 n) !}=\frac{x}{\sin x} .
$$

This case was known to Gessel and Viennot [3, §11]. The number $f^{\lambda / \mu}$ is not affected by conjugating (transposing) the shape. Baryshnikov and Romik [1, Thm. 1] consider the conjugate shape $\sigma(3,3,2, n)^{\prime}$, and their result for this shape is equivalent to equation (2.1). They state their generating function as $x(\cot (x / 2)-\cot (x))$, and not in the simpler form $x / \sin (x)$. Let us note, however, that the more general shapes considered by Baryshnikov and Romik are not covered by our results. It would be interesting to find a common generalization. In principle the technique of Baryshnikov and Romik applies to any shape $\sigma(a, b, c, n)$, but the computations become very complicated.

\section{A generalization of $\zeta(2), \zeta(4)$ and $\zeta(6)$}

The Riemann zeta function is defined by $\zeta(s)=\sum_{n \geq 1} n^{-s}$ for $\Re(s)>1$. It is well-known that if $k$ is a positive integer then

$$
\zeta(2 k)=(-1)^{k+1} \frac{B_{2 k}(2 \pi)^{2 k}}{2(2 k) !},
$$


where $B_{2 k}$ denotes a Bernoulli number. Note that

$$
\zeta(2 k)=p_{1}\left(1,1 / 2^{2 k}, 1 / 3^{2 k}, \ldots\right)=p_{k}\left(1,1 / 2^{2}, 1 / 3^{2}, \ldots\right),
$$

where $p_{j}=\sum x_{i}^{j}$, the $j$ th power sum symmetric function. This formula suggests looking at $f\left(1,1 / 2^{2 k}, 1 / 3^{2 k}, \ldots\right)$ for other symmetric functions $f$. In principle there is no problem: expand $f$ in terms of power sums, say $f=\sum_{\lambda} c_{\lambda} p_{\lambda}$. Then

$$
f\left(1,1 / 2^{s}, 1 / 3^{s}, \ldots\right)=\sum_{\lambda} c_{\lambda} \zeta\left(\lambda_{1} s\right) \zeta\left(\lambda_{2} s\right) \cdots \zeta\left(\lambda_{\ell} s\right)
$$

where $\lambda=\left(\lambda_{1}, \ldots, \lambda_{\ell}\right)$ with $\lambda_{1} \geq \cdots \geq \lambda_{\ell}>0$. In particular, if $f$ is homogeneous of degree $n$ then $f\left(1,1 / 2^{2 k}, 1 / 3^{3 k}, \ldots\right)=\alpha \pi^{2 k n}$, where $\alpha \in \mathbb{Q}$. See Hoffman [4]. We may ask, however, whether there is a simple description of $\alpha$ for a suitable choice of $f$. Here we consider the case where $f=s_{\lambda / \mu}$, a skew Schur function. We will give a simple expression for $s_{\lambda / \mu}\left(1,1 / 2^{2 k}, 1 / 3^{3 k}, \ldots\right)$ for $k=1,2,3$ in terms of the number $f^{\sigma / \tau}$ of SYT of a certain skew shape $\sigma / \tau$. The proof breaks down for $k \geq 4$, and there does not seem to be an analogous formula in this case.

The basic idea is to expand $s_{\lambda / \mu}$ as a polynomial in the elementary symmetric functions $e_{j}$, then to evaluate $e_{j}\left(1,1 / 2^{k}, 1 / 3^{k}, \ldots\right)$ and interpret the resulting expression in terms of $f^{\sigma / \tau}$. The expansion of $s_{\lambda / \mu}$ in terms of $e_{j}$ 's is given by the dual Jacobi-Trudi identity (1.3), so we next turn to the evaluation of $e_{j}\left(1,1 / 2^{2 k}, 1 / 3^{2 k}, \ldots\right)$ for $k=1,2,3$.

Lemma 3.6. We have:

(a)

$$
e_{j}\left(1,1 / 2^{2}, 1 / 3^{2}, \ldots\right)=\frac{\pi^{2 j}}{(2 j+1) !}
$$

(b)

$$
e_{j}\left(1,1 / 2^{4}, 1 / 3^{4}, \ldots\right)=\frac{2^{2 j+1} \pi^{4 j}}{(4 j+2) !}
$$

(c)

$$
e_{j}\left(1,1 / 2^{6}, 1 / 3^{6}, \ldots\right)=\frac{6 \cdot 2^{6 j} \pi^{6 j}}{(6 j+3) !} .
$$

Proof. (a) The Weierstrass product for the function $\sin (\pi z) / \pi z$ takes the form

$$
\frac{\sin (\pi z)}{\pi z}=\prod_{k \geq 1}\left(1-\frac{z^{2}}{k^{2}}\right) .
$$

Consider the Taylor series expansion at $z=0$. On the one hand, the coefficient of $z^{2 j}$ in $\sin (\pi z) / \pi z$ is $(-1)^{j} \pi^{2 j} /(2 j-1)$ !. On the other hand, by equation $(3.1)$ this coefficient is $(-1)^{j} e_{j}\left(1,1 / 2^{2}, 1 / 3^{2}, \ldots\right)$, and the proof follows. This result was known to Euler [2], with essentially the same proof (though without sufficient rigor). 


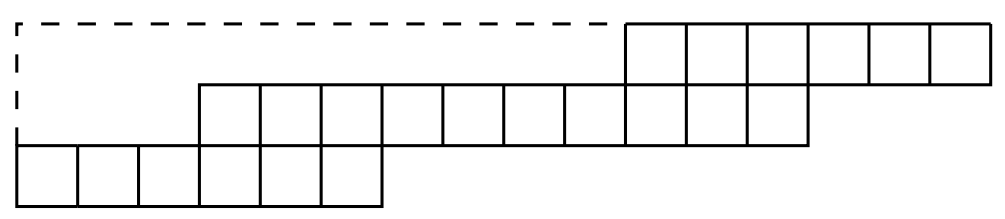

Figure 2: The skew shape $\left(4(2,2,1)+2 \gamma_{3}+3 \delta_{3}\right) /\left(4(1,0,0)+3 \delta_{3}\right)$

(b) We have

$$
\begin{aligned}
\prod_{k \geq 1}\left(1-\frac{z^{4}}{k^{4}}\right) & =\prod_{k \geq 1}\left(1-\frac{z^{2}}{k^{2}}\right)\left(1+\frac{z^{2}}{k^{2}}\right) \\
& =\frac{\sin (\pi z) \frac{\sin (\pi i z)}{\pi z}}{\pi i z} \\
& =\frac{\cos ((1-i) \pi z)-\cos ((1+i) \pi z)}{2 \pi^{2} i z^{2}} \\
& =\sum_{n \geq 0}(-1)^{j} 2^{2 j+1} \pi^{4 j} \frac{z^{4 j}}{(4 j+2) !}
\end{aligned}
$$

from which the proof follows.

(c) Let $\omega=e^{2 \pi i / 3}$. We have

$$
\begin{aligned}
\prod_{k \geq 1}\left(1-\frac{z^{6}}{k^{6}}\right) & =\prod_{k \geq 1}\left(1-\frac{z^{2}}{k^{2}}\right)\left(1-\frac{\omega z^{2}}{k^{2}}\right)\left(1-\frac{\omega^{2} z^{2}}{k^{2}}\right) \\
& =\frac{\sin \pi z}{\pi z} \cdot \frac{\sin \pi \omega z}{\pi \omega z} \cdot \frac{\sin \pi \omega^{2} z}{\pi \omega^{2} z} \\
& =-\frac{1}{4 \pi^{3} z^{3}}\left(\sin (2 \pi z)+\sin (2 \pi \omega z)+\sin \left(2 \pi \omega^{2} z\right)\right) \\
& =\sum_{j \geq 0}(-1)^{j} 6 \cdot 2^{6 j} \pi^{6 j} \frac{z^{6 j}}{(6 j+3) !},
\end{aligned}
$$

from which the proof follows.

Let $\lambda$ and $\mu$ be partitions of length at most $m$. Let $\delta_{m}=(m-1, m-2, \ldots, 0)$ and $\gamma_{m}=\left\langle 1^{m}\right\rangle$, the partition with $m$ parts equal to 1 . For instance, if $\lambda=(2,2,1)$ and $\mu=(1,0,0)$ then $4 \lambda+2 \gamma_{2}+3 \delta_{3}=(16,13,6)$ and $4 \mu+3 \delta_{3}=(10,3,0)$. The diagram of the skew shape $\left(4 \lambda+2 \gamma_{3}+3 \delta_{3}\right) /\left(4 \mu+3 \delta_{3}\right)$ is shown in Figure 2 .

Theorem 3.7. For any skew shape $\lambda / \mu$ where $\lambda_{1} \leq m$ and $|\lambda / \mu|=n$, the following formulas hold.

(a) $s_{\lambda / \mu}\left(1,1 / 2^{2}, 1 / 3^{2}, \ldots\right)=\frac{\pi^{2 n} f^{\left(2 \lambda^{\prime}+\gamma_{m}+\delta_{m}\right) /\left(2 \mu^{\prime}+\delta_{m}\right)}}{(2 n+m) !}$ 
(b) $s_{\lambda / \mu}\left(1,1 / 2^{4}, 1 / 3^{4}, \ldots\right)=\frac{2^{m+2 n} \pi^{4 n} f^{\left(4 \lambda^{\prime}+2 \gamma_{m}+3 \delta_{m}\right) /\left(4 \mu^{\prime}+3 \delta_{m}\right)}}{(4 n+2 m) !}$

(c) $s_{\lambda / \mu}\left(1,1 / 2^{6}, 1 / 3^{6}, \ldots\right)=\frac{6^{m} 2^{6 n} \pi^{6 n} f^{\left(6 \lambda^{\prime}+3 \gamma_{m}+5 \delta_{m}\right) /\left(6 \mu^{\prime}+5 \delta_{m}\right)}}{(6 n+3 m) !}$

Proof. (a) By equation (1.3) and Lemma 3.6(a) we have

$$
\begin{aligned}
s_{\lambda / \mu}\left(1,1 / 2^{2}, 1 / 3^{2}, \ldots\right) & =\operatorname{det}\left[e_{\lambda_{i}^{\prime}-\mu_{j}^{\prime}-i+j}\left(1,1 / 2^{2}, 1 / 3^{2}, \cdots\right)\right]_{i, j=1}^{m} \\
& =\operatorname{det}\left[\pi^{2\left(\lambda_{i}^{\prime}-\mu_{j}^{\prime}-i+j\right)} /\left(2\left(\lambda_{i}^{\prime}-\mu_{j}^{\prime}-i+j\right)+1\right) !\right]_{i, j=1}^{m} \\
& =\pi^{2 n} \operatorname{det}\left[1 /\left(2\left(\lambda_{i}^{\prime}-\mu_{j}^{\prime}-i+j\right)+1\right) !\right]_{i, j=1}^{m} .
\end{aligned}
$$

Now

$$
\begin{aligned}
2\left(\lambda_{i}^{\prime}-\mu_{j}^{\prime}-i+j\right)+1 & =\left(2 \lambda_{i}^{\prime}-2 \mu_{j}^{\prime}+1+(m-i)-(m-j)\right)-i+j \\
& =\left(2 \lambda^{\prime}+\gamma_{m}+\delta_{m}\right)_{i}-\left(2 \mu^{\prime}+\delta_{m}\right)_{j}-i+j,
\end{aligned}
$$

and the proof follows from equation (1.2).

(b) Similarly to (a) we have

$$
\begin{aligned}
s_{\lambda / \mu}\left(1,1 / 2^{4}, 1 / 3^{4}, \ldots\right)= & \operatorname{det}\left[e_{\lambda_{i}^{\prime}-\mu_{j}^{\prime}-i+j}\left(1,1 / 2^{4}, 1 / 3^{4}, \cdots\right)\right]_{i, j=1}^{m} \\
= & \operatorname{det}\left[2^{2\left(\lambda_{i}^{\prime}-\mu_{j}^{\prime}-i+j\right)+1} \pi^{4\left(\lambda_{i}^{\prime}-\mu_{j}^{\prime}-i+j\right)} /\right. \\
& \left.\left(4\left(\lambda_{i}^{\prime}-\mu_{j}^{\prime}-i+j\right)+2\right) !\right]_{i, j=1}^{m} \\
= & 2^{2 n+m} \pi^{2 n} \operatorname{det}\left[1 /\left(4\left(\lambda_{i}^{\prime}-\mu_{j}^{\prime}-i+j\right)+2\right) !\right]_{i, j=1}^{m} .
\end{aligned}
$$

Now

$$
\begin{aligned}
4\left(\lambda_{i}^{\prime}-\mu_{j}^{\prime}-i+j\right)+2 & =\left(4 \lambda_{i}^{\prime}-4 \mu_{j}^{\prime}+2+3(m-i)-3(m-j)\right)-i+j \\
& =\left(4 \lambda^{\prime}+2 \gamma_{m}+3 \delta_{m}\right)_{i}-\left(4 \mu^{\prime}+3 \delta_{m}\right)_{j}-i+j,
\end{aligned}
$$

and the proof follows from equation (1.2).

(c) Analogous to (b).

It is natural to ask about $k \geq 4$, i.e., the evaluation of $s_{\lambda / \mu}\left(1,1 / 2^{2 k}, 1 / 3^{2 k}, \ldots\right)$ for $k \geq 4$. Our method appears to break down. The trigonometric identities needed for $k=2,3$ do not seem to have an analogue for $k \geq 4$. Indeed, $e_{j}\left(1,1 / 2^{8}, 1 / 3^{8}, \ldots\right)$ does not have the form $a(j) \pi^{16 j}$ where $a(j)$ is a rational number whose numerator and denominator can be factored into small primes. Also a problem is the case $e_{1}\left(1,1 / 2^{2 k}, 1 / 3^{2 k}, \ldots\right)$, since in general there is no simple formula for Bernoulli numbers. We can still ask, however, whether there is a simple combinatorial interpretation of $s_{\lambda / \mu}\left(1,1 / 2^{2 k}, 1 / 3^{2 k}, \ldots\right)$ after suitable normalization. 


\section{References}

[1] Y. Baryshnikov and D. Romik, Enumeration formulas for Young tableaux in a diagonal strip, Israel J. Math. 178 (2010), 157-186.

[2] L. Euler, De summis serierum reciprocarum, Commentarii academiae scientiarum Petropolitanae 7 (1740), 123-134; Opera Omnia, Series 1, volume 14, pp. 73-86.

[3] I. M. Gessel and X. G. Viennot, Determinants, paths, and plane partitions, preprint, 1989.

[4] M. E. Hoffman, A character on the quasi-symmetric functions coming from multiple zeta values, Electronic J. Combinatorics 15(1) (2009), R97.

[5] R. Stanley, Enumerative Combinatorics, vol. 1, Wadsworth and Brooks/Cole, Pacific Grove, CA, 1986; second printing, Cambridge University Press, New York/Cambridge, 1996.

[6] R. Stanley, Enumerative Combinatorics, vol. 2, Cambridge University Press, New York/Cambridge, 1999 